\title{
Outbreak of peracute Glässer's disease in suckling piglets in a self-recruiting high health farrow to grower herd: A Case report
}

Odd Magne Karlsen ( $\sim$ odd.magne.karlsen@nortura.no )

Resident of Norwegian University of Life Sciences (NMBU)

\section{Case report}

Keywords: Glässer's disease, pig, per-acute disease

Posted Date: December 7th, 2020

DOl: https://doi.org/10.21203/rs.3.rs-121032/v1

License: (c) (i) This work is licensed under a Creative Commons Attribution 4.0 International License.

Read Full License 


\section{Abstract}

\section{Background}

Glässer's disease is caused by a bacterial infection where the causative agent is Glaesserella parasuis and is of rising concern in Norwegian high health pig herds. Different factors can trigger the onset of Glässer's disease, including management practices such as unstable room temperature, poor ventilation or impaired immunity. Outbreaks of peracute Glässer's disease in suckling piglets are rarely described.

\section{Case presentation}

In a high health, farrow to grower pig herd 270 suckling piglets out of 920 died in a peracute to acute manner during a 20-day period. Post-mortem examinations revealed findings consistent with congestion and oedema and histopathological examination found no signs of inflammation or intravascular microthrombi. Bacteriological examination detected G. parasuis in relevant tissues. Emesis in half of the piglets and central nervous signs in a few was found after first intramuscular injection of procaine penicillin. Anaemia, poor temperature regulation in the farrowing unit and poor hygiene were factors contributing to the disease outbreak.

\section{Conclusions}

In the present case, it was concluded that an outbreak with high mortality and predominantly peracute to acute clinical disease in 3-5 week old suckling piglets was caused by Glässer's disease (G. parasuis).

\section{Background}

Glässers disease is characterised by fibrinous polyserositis and arthritis in pigs and the etiological agent is the gram-negative bacteria Glaesserella parasuis (1) formerly known as Haemophilus parasuis, a member of the family Pasteurellaceae. It is a fastidious bacterium with heterogenous phenotypic and genotypic traits, including virulence factors $(2,3)$.

Clinical signs are mainly observed in 4- to 8-week-old pigs, although the age of affected animals may vary depending on the level of acquired maternal immunity and colonization (4). Glässer's disease outbreaks have been described in adult pigs originating from naïve herds, usually after comingling with healthy G. parasuis-colonized animals (5). The incubation period varies depending on the infecting strain and ranges from less than 24 hours to $4-5$ days post inoculation (6). Peracute disease may result in sudden death without characteristic gross lesions (7). The typical clinical signs of acute Glässer's disease include high fever $\left(41,5^{\circ} \mathrm{C}\right)$, coughing, abdominal breathing, swollen joints with lameness, and central nervous signs such as lateral recumbency, paddling, and trembling (8-11). These signs may be seen jointly or independently. Animals with mild to moderate clinical signs usually survive the acute phase of the disease and may develop chronic signs characterized by rough hair, reduced growth rate, and 
lameness (4). Morbidity and mortality rates associated with Glässer's disease in affected farms may vary from $5-10 \%$ in conventional herds to $75 \%$ in naïve herds (5).

G. parasuis colonize the upper respiratory tract of healthy pigs, and the detection of this microorganism in the nasal cavity and trachea does not imply disease development. Systemic isolates are more likely to carry important virulence factors and, therefore, those isolates should be pursued for laboratory conformation of morbidity and mortality caused by this agent (2).

Peracutely diseased pigs usually die without characteristic gross lesions, but may show petechial hemorrhages in some tissues $(7,10)$. Histologically, these pigs show septicemia-like microscopic lesions such as disseminated intravascular coagulation and microhemorrhages (12).

Vaccination and antibiotics can be used to prevent and control G. parasuis infections. In some countries like Norway, prophylactic use of antibiotics is restricted. These drugs are only allowed for metaphylactic and therapeutic purposes. Individual parenteral treatments tend to be more effective than water or feed medication, since pigs clinically affected by $G$. parasuis infection are prostrate and less likely to ingest the required antibiotic dose via food or water (2).

The aim of the present case report is to describe an acute/peracute outbreak of Glässer's disease affecting suckling piglets in a Norwegian specific pathogen free (SPF) herd.

\section{Case Presentation}

The author of this case report was contacted by a pig herd owner due to a sudden onset of peracute/acute mortality in 2-3-week-old suckling piglets the previous day (day 1). Due to Easter holiday, no veterinary diagnostic laboratories were open for submission of samples until one week later.

The herd was a specific pathogen free (SPF) sow herd producing growers, $25-35 \mathrm{~kg}$, for finisher farms. It ran a $5 \frac{1}{2}$ weeks batch system based on a sow cycle of 22 weeks (116 days gestation 33 days lactation and 5 days from weaning to insemination) with four batches, each batch comprising approximately 100 sows. The farm has one farrowing unit (containing one room) with 98 farrowing pens and one unit for all the weaners and growers. In the case batch, the farrowing room held 83 sows, eight of them were first parity sows, and 920 piglets. All piglets were weaned and moved to the grower unit on day 14 .

The herd was self-recruiting and produced its own breeding sows. No pigs from other farms had been introduced to the herd since a depopulation and repopulation of pigs were completed 3 years ago.

SPF-herds in Norway are required to document absence of Actinobacillus pleuropneumonia, Brachyspira hyodysenteria and toxin producing Pasturella multocida. In addition, the commercial Norwegian pig population as a whole has documented freedom from Mycoplasma hyopneumonia, porcine reproductive and respiratory syndrome virus (PRRSV), swine influenza virus (SIV) except the pandemic H1N1 virus, Aujezkys disease, transmissible gastroenteritis virus (TGEV), porcine epidemic diarrhoea (PED) and at that time, porcine respiratory coronavirus (PRCV) through active serosurveillance (13). 
Erysipelas, parvo virus and E. coli vaccinations of the sows were performed according to manufacturer's recommendations. According to herd health records, the farm had low use of antimicrobials, with treatments only initiated on medical indication and administered as individual pig treatments.

The herd was visited by an on-call veterinarian the same day of the call (day 2). It was noted that approximately 30 piglets had died. No piglets showing clinical signs of disease were found. Four piglets were examined post-mortem (PME) on farm with scarce findings and without reaching any conclusions. During the evening and following night, 80 more piglets had died. The next morning (day 3), a new herd visit were organized with the on-call veterinarian and the author. Piglets and sows showing clinical signs of disease were identified.

The clinical evaluation was limited in time due to the extent and peracute nature of the disease. Single piglets in each pen, lied down in sternal recumbency, with a rapid, superficial breathing. Within minutes affected piglets rolled over in lateral recumbency, did not respond to tactile and vocal stimuli and died. Affected piglets were evenly distributed throughout the pens in the farrowing room, however their littermates appeared unaffected and healthy

Four out of 83 lactating sows had rectal temperatures above $40^{\circ} \mathrm{C}$. They had problems rising, ataxia and had excessive and high-pitched vocalisation. One sow died later the same day.

On farm PME were performed on piglets which had died the night before. A preliminary gross pathology status was pale musculature, serosanguinous fluid in the peritoneal cavity and pericardial sac, fine threads of fibrin over the intestines (Figure 1). Some piglets had intestinal haemorrhages and petechial bleedings in the kidneys.

Based on clinical findings and gross pathology bacterial septicaemias or viruses able to induce acute/peracute disease and high mortality was suspected. A presumptive list of differential diagnoses contained Glaesserella parasuis (Glässer's disease), Erysipelothrix rhusiopathiae (erysipelas), oedema disease caused by beta-hemolytic Shiga toxin 2e -positive Escherichia coli and Streptococcus suis, classical swine fever virus (CSF) and african swine fever virus (ASF).

Contact was made to the Norwegian Food Safety Authority (NFSA) as the competent authority and the national reference laboratory for CSF and ASF , the Norwegian Veterinary Institute (NVI) informing them of the clinical and preliminary gross pathology findings and that notifiable diseases like classical swine fever and african swine fever were on the differential diagnosis list.

On farm PMEs were performed on altogether 15 piglets. Samples for bacteriologic examination were collected and frozen, including 5 heads, 5 livers, 4 lungs, 1 small intestine and kidneys from five piglets. Samples from heart, lung, liver, kidney and brain from three piglets were collected and fixated on formaldehyde for histopathological evaluation. 
Additional herd visits, including clinical evaluations, on farm PME and sampling for laboratory examination were performed on day 4, 10 and 20. Three intact dead piglets and formaldehyde fixated samples from heart and lymph nodes from two piglets were collected on day 4. Six fresh livers and spleens were sampled on day 20.

Blood samples from 10, 5 and 11 piglets were collected on day 4, 10 and 20 respectively. The blood was collected in vacutainers containing ethylenediamine tetra-acetic acid (EDTA) as an anticoagulant. A hematologic profile was obtained from a local lab.

Collection of data regarding management routines, biosecurity and environmental factors were performed in on-farm interviews with the workers on the farm.

The piglets died in three rounds with the highest mortality rates during the first three days (Figure 2).

The most consistent gross pathological findings were serous, protein rich fluids in the peritoneal cavity and in the pericardium, fine threads of fibrin covering the abdominal organs, anaemia and swollen livers and spleens, while the histopathological findings were congestion and oedema in many organs, and an increased haemopoietic activity in the livers and spleens. Six and three formalin-fixed lung tissue samples were immunohistochemistry negative for influenza A virus nucleoprotein and porcine circovirus type 2 (PCV2), respectively.

The bacteriologic examination found G. parasuis in 12 out of 14 samples originating from the frozen specimens collected at day 3. G. parasuis was also isolated in 11 out of 12 of the fresh livers and spleens sampled on day 20. Streptococcus $s p$ was isolated in 2 samples and Strept suis serotype 8 and Strept suis in one each, of the frozen samples. In the fresh livers and spleens there were also found Aerococcus sp in 7 out of 12 samples. Two of the G. parasuis isolates from fresh livers and spleens were typed as serotype 9 based on indirect hemagglutination test (IHA) performed at the Innovative Veterinary Diagnostic laboratory (IVD GmgH, Hannover, Germany).

Low blood cell counts, and a low haemoglobin was found in the blood collected on day 4, confirming anaemia.

The interviews revealed that iron paste given to the piglets within the first 24 hours after farrowing was the only routine iron supplementation for piglets on the farm. The automatic regulation of the ventilation system of the farrowing room didn't work and was handled manually. There were no routines for washing and disinfection between farrowing batches.

Given the per-acute progression of disease, the high mortality and no expected diagnostic laboratory answers due to Easter holiday, a metaphylactic treatment was initiated on day 3 . This was also deemed relevant to provide diagnostic information. Half of the piglets (group 1) received intramuscular injections (IM) of $600 \mathrm{mg}$ benzylpenicillin procaine $\mathrm{q} 24 \mathrm{~h}$ for three days. Following penicillin treatment, no piglets died after 12 hours, whereas 50 piglets died during the following night in the untreated group (group 2). Group 2 were then given the same antibiotic treatment on day 4 . 
After a second rise in piglet mortality on day 9, all piglets were IM injected with $600 \mathrm{mg}$ benzylpenicillin procaine $\mathrm{q} 24 \mathrm{~h}$ for five days. The piglet mortality stopped within twelve hours after the first injections with benzylpenicillin procaine every time a new treatment was initiated (Figure 2).

A daily routine supplying the piglets with iron-supplemented peat on the floor for oral consumption from day 6 and onwards until weaning was established (Figure 2).

The sows with signs of polyarthritis and meningitis were treated with an IM injection of 10,5 g benzylpenicillin procaine q $24 \mathrm{~h}$ in three days and a single IM injection of $800 \mathrm{mg}$ ketoprofen. The treated sows had no clinical signs of disease the day after initiated treatment and had no relapse of disease.

Approximately half of the piglets getting their first injection of benzylpenicillin procaine on day 3 and 4 started vomiting within an hour after injection. A few showed central nervous signs, lying in lateral recumbency, with paddling movements of the hind limbs. After a few hours, all of them appeared healthy. None of these died and the next day it was not possible to see which ones had been sick. This was not seen again in the subsequent treatment.

\section{Discussion And Conclusions}

Different factors can trigger the onset of Glässer's disease, including management practices such as unstable room temperature, poor ventilation or early weaning (2).

There was a change in weather and metrological data documented a drop in the middle temperature from $0^{\circ} \mathrm{C}$ to $-3^{\circ} \mathrm{C}$ a few days before the first outbreak. The ventilation system in the farrowing room was manually operated and was not able to automatically adjust for temperature drops.

There were no established cleaning and disinfection routines between batches in the farrowing room.

There has been reported an epidemiological association between G. parasuis infection and PRRSV, PCV2 and SIV type A $(22,23)$. There is no PRRSV in Norwegian pigs and there were no clinical signs or laboratory results indicating PCV2 or influenza A infection.

The clinical findings of a per-acute disease were confirmed by the pathologic findings. The histopathological examination found no signs of organisation of the disease process and no signs of inflammation. The exception being the increased haemopoietic activity that was found in the liver and spleen. The anaemia found in the blood samples confirmed the anaemia found in the PMEs. Only supplementing iron paste to the piglets the day after farrowing might provide enough iron for 1 week in new-born piglets (16). Piglets are born with a very limited iron storage in their livers and sow milk contains little iron, this makes them vulnerable to develop iron deficiency and anaemia. The increased haemopoietic activity also makes sense in concluding that anaemia in the piglets was present before the disease outbreak. Anaemia is known to impair immunity (2) and may have contributed to the disease outbreak. 
The findings of polyserositis and polyarthritis in the piglets in the on-farm PME on day 20 might indicate that there was little specific immunity for the prevalent $G$. parasuis strain in the start of the outbreak, but more so 3 weeks after. The piglets seem to be naïve, having no maternal antibodies to the diseasecausing strain or heterological strains capable of inducing cross protection. The reservoir for $G$. parasuis is pigs and as the farm is self-recruiting its own gilts, introductions of new serotypes to the herd from pigs are not likely. With only eight first parity sows, most of the sows were second and third parity sows, the maternal immune status for herd pathogens and commensals should be better than with a high first parity sow ratio. In a healthy scenario, colonization by G. parasuis develops when piglets are still protected by maternal immunity and a balance between colonization and immunity occurs (2). There was no evident breach in the biosecurity on the farm. This may indicate that there was a very low maternal immunity against a pathogenic strain already present in the herd or an unknown new introduction of a $G$. parasuis strain of which the herd was naïve.

The dosage of penicillin procaine used in the treatments is quite high for pigs around $5 \mathrm{~kg}$, but penicillin procaine is considered a safe antibiotic with high therapeutic index and few side effects. G. parasuis might have induced endothelial damage to such extent in the first outbreak that it allowed procaine through the blood brain barrier, reaching the brain tissue, and causing the adverse effects.

The clinical signs and lesions described for G. parasuis systemic infection are not pathognomonic and several differential diagnosis caused by other agents need to be excluded (2). ASF and CSF cannot be diagnosed based on clinical signs or gross lesions $(14,15)$, so samples for laboratory examination were sent to the national reference laboratory NVI. The prompt positive outcome of the initiated antibiotic treatments made ASF and CSF a much less likely differential diagnosis.

The first briefings from the laboratory on bacteriology results arrived on day 14 . The split treatment strengthened the conclusion of a bacterial sensitive to penicillin as the cause. It made Oedema disease a less likely differential diagnosis list. It also made it easier in the recurring treatments to keep using a narrow spectrum antibiotic with a short subtherapeutic concentration time tail in the pigs.

The consistent bacteriological finding of $G$. parasuis in the frozen samples (day 3 ) and the fresh samples (day 20) from systemic organs including brain, liver and spleen indicates that $G$. parasuis may play a role in the development of the disease.

Streptococcus suis usually affects pigs at the same age as $G$. parasuis and may cause similar lesions $(17,18)$. Some of the findings of Streptococcus suis and Strept. sp indicate a coinfection with $G$. parasuis. Due to their limited isolation and their lack of serotyping consistency it was considered not to be the main cause of this peracute outbreak.

Aerococcus sp. as $A$. viridans and $A$. suis sp. nov. has been isolated from lungs, joints, gut and brain in pigs with pneumonia, arthritis, enteritis and meningitis respectively $(19,20)$. Aerococcus sp.was only found in samples taken on day 20. With little knowledge of the pathogenic potential of Aerococcus $s p$, it is difficult to assess its role in the development of the disease in the current case. 
There were found no other signs of CSF, ASF, erysipelas or oedema disease in the piglets and samples.

Glaesserella parasuis serotypes $1,5,10,12,13$ and 14 are known to cause mortality in pigs. In spite of presence of many different strains within a herd, usually one single prevalent strain is associated with an disease outbreak (21). Glaesserella parasuis serotype 9 is found in healthy pigs (3). Severity of disease depends on the virulence of the $G$. parasuis strain, the immunity of the piglet, the concomitant presence of other pathogens in the herd, and the genetic resistance of the host (2).

Glaesserella parasuis is present in most, if not all Norwegian pig farms. A serological screening of 5176 blood samples from pigs in 113 nucleus or multiplier herds at the end of the 1980s, found G. parasuis antibodies in pigs from $95 \%$ of the herds (24). This prevalence is likely similar today. It is poorly documented which serotypes G. parasuis the Norwegian pigs have.

It was concluded that $G$. parasuis caused the per-acute disease in 3-5 weeks old suckling piglets with a mortality reaching $30 \%$. PME revealed findings consistent with congestion and oedema and histopathological examination found no signs of inflammation or intravascular microthrombi. Emesis in half of the piglets and central nervous signs in a few was found after first IM injection of procaine penicillin. Anaemia, poor temperature regulation in the farrowing unit and poor hygiene were found to be triggering factors for the disease.

\section{Abbreviations}

Glaesserella parasuis - G. parasuis

Specific pathogen free - SPF

Porcine reproduction and respiratory syndrome virus - PRRSV

Swine influenza virus - SIV

Transmissible gastroenteritis virus - TGEV

Porcine epidemic diarrhoea - PED

Porcine respiratory coronavirus - PRCV

Post-mortem examination - PME

Classical swine fever virus - CSF

African swine fever virus - ASF

Norwegian Food Safety Authority - NFSA

Norwegian Veterinary Institute - NVI 
Ethylenediamine tetra-acetic acid - EDTA

Porcine circovirus type 2 - PCV2

Indirect hemagglutination test - IHA

Intramuscular injections - IM

Milligram - mg

Every - q

hour- $\mathrm{h}$

Gram - g

\section{Declarations}

\section{Ethics approval and consent to participate}

The farmer gave his informed consent to participate.

\section{Consent for publication}

The farmer gave his informed consent to publish the results from the analyses of samples and measures taken from their farms.

\section{Availability of data and material}

The data generated for the current case report are kept and stored by the corresponding author. The data are available from the corresponding author on reasonable request.

\section{Competing interests}

The author declare that he have no competing interests.

\section{Funding}

Not applicable

\section{Authors contributions}

OMK drafted the manuscript. OMK did farm visits, on farm PMEs and collected most of the samples.

\section{Acknowledgements}


The Author would like to acknowledge my patient supervisor and the pathologists and bacteriologists at the Norwegian Veterinary Institute. He would also acknowledge Marianne Oropeza Moe, Carl Andreas Grøntvedt and Stine Gulliksen for help with the manuscript.

\section{Authors' information (optional)}

\section{References}

1. Dickerman A, Bandara AB, Inzana TJ. Phylogenomic analysis of haemophilus parasuis and proposed reclassification to glaesserella parasuis, gen. Nov., comb. nov. Int J Syst Evol Microbiol. 2020 Oct 8;70(1):180-6.

2. Aragon V, Segalés J, Tucker AW (Dan). Glässer's Disease. In: Zimmerman JJ, Karriker LA, Ramirez A, Schwartz KJ, Stevenson GW, Zhang J, editors. Diseases of Swine 11th edition. Wiley-Blackwell; 2019. p. 844-53.

3. Kielstein T, Rapp-Danielson VJ. Designation of 15 serovars of Haemophilus parasuis on the basis of immunodiffusion using heat-stable antigen extracts. J Clin Microbiol [Internet]. 1992 [cited 2012 Aug 2];30(4):862-5. Available from: http://jcm.asm.org/content/30/4/862.short

4. Aragon V, Segalés J, Oliveira S. Glässer's Disease. In: Zimmerman JJ, Karriker LA, Ramirez A, Schwartz KJ, Stevenson GW, editors. Diseases of Swine. 10th ed. Ames (Iowa, USA): Wiley-Blackwell; 2012. p. 760-9.

5. Wiseman B, Harris B, Glock R, Wilkins L. Management of seedstock that is negative for Haemophilus parasuis. Proc Annu Am Assoc Swine Pract. 1989;23-5.

6. Aragon V, Cerdà-Cuéllara M, Frailea L, Mombargc M, Nofraríasa M, Olveraa A, et al. Correlation between clinico-pathological outcome and typing of Haemophilus parasuis field strains. Vet Microbiol [Internet]. 2010 May 19 [cited 2012 Aug 2];142(3-4):387-393. Available from: http://www.ncbi.nlm.nih.gov/pubmed/19945233

7. Peet RL, Fry J, Lloyd J, Henderson J, Curran J, Moir D. Haemophilus parasuis septicaemia in pigs. Aust Vet J [Internet]. 1983 Mar 10 [cited 2012 Jul 30];60(6):187-187. Available from: http://doi.wiley.com/10.1111/j.1751-0813.1983.tb05960.x

8. Neil D, McKay K, L'Ecuyer C, Corner A. Glasser's disease of swine produced by the intratracheal inoculation of Haemophilus suis. Can J Comp Med [Internet]. 1969 [cited 2012 Jul 30];33(3):187-93. Available from: http://www.ncbi.nlm.nih.gov/pmc/articles/PMC1319372/

9. Nielsen R, Danielsen V. An outbreak of Glasser's disease. Studies on etiology, serology and the effect of vaccination. Nord Vet Med [Internet]. 1975 [cited 2012 Jul 30];27(1):20-5. Available from: http://ukpmc.ac.uk/abstract/MED/1114045

10. Riley MG, Russell EG, Callinan RB. Haemophilus parasuis infection in swine. J Am Vet Med Assoc [Internet]. 1977 [cited 2012 Jul 30];171(7):649-51. Available from: http://ukpmc.ac.uk/abstract/MED/914694 
11. Vahle JL, Haynes JS, Andrews JJ. Experimental reproduction of Haemophilus parasuis infection in swine: clinical, bacteriologic, and morphologic findings. J Vet Diagnostic Investig [Internet]. 1995 [cited 2012 Jul 30];7(4):476-80. Available from: http://vdi.sagepub.com/content/7/4/476.short

12. Amano H, Shibata M, Kajio N, Morozumi T. Pathologic observations of pigs intranasally inoculated with serovar 1, 4 and 5 of Haemophilus parasuis using immunoperoxidase method. J Vet Med Sci [Internet]. 1994 [cited 2012 Jul 31];56(4):639-44. Available from: http://ukpmc.ac.uk/abstract/MED/7999883

13. Grøntvedt CA, Bang Nordstoga A, Hopp P. The surveillance programme for specific viral infections in swine herds in Norway 2019. 2020.

14. Kirkland PD, Potier M-F Le, Finlaison D. Pestiviruses. In: Zimmerman JJ, Karriker LE, Ramirez A, Schwartz KJ, Stevenson GW, Zhang J, editors. Diseases of Swine 11th edition. 11th ed. Hoboken: Wiley-Blackwell; 2019. p. 622-40.

15. Sánchez-Vizcaíno JM, Laddomada A, Arias ML. African Swine Fever Virus. In: Zimmerman JJ, Karriker LE, Ramirez A, Schwartz KJ, Stevenson GW, Zhang J, editors. Diseases of Swine 11th edition. 11th ed. Hoboken: Wiley-Blackwell; 2019. p. 443-52.

16. Svoboda M, Píšt'ková K. Oral iron administration in suckling piglets - a review. Acta Vet Brno. 2018;87(1):77-83.

17. Reams RY, Glickman LT, Harrington DD, Thacker HL, Bowersock TL. Streptococcus Suis Infection in Swine: A Retrospective Study of 256 Cases. Part II. Clinical Signs, Gross and Microscopic Lesions, and Coexisting Microorganisms. J Vet Diagnostic Investig [Internet]. 1994 Jul 1 [cited 2015 Feb 9];6(3):326-34. Available from: http://vdi.sagepub.com/content/6/3/326.short

18. Vecht U, Arends JP, van der Molen EJ, van Leengoed LA. Differences in virulence between two strains of Streptococcus suis type II after experimentally induced infection of newborn germ-free pigs. Am J Vet Res [Internet]. 1989 Jul 1 [cited 2015 Jan 19];50(7):1037-43. Available from: http://europepmc.org/abstract/med/2774320

19. Martín V, Vela Al, Gilbert M, Cebolla J, Goyache J, Domínguez L, et al. Characterization of Aerococcus viridans isolates from swine clinical specimens. J Clin Microbiol. 2007;45(9):3053-7.

20. Vela Al, García N, Latre M V., Casamayor A, Sánchez-Porro C, Briones V, et al. Aerococcus suis sp. nov., isolated from clinical specimens from swine. Int J Syst Evol Microbiol. 2007;57(6):1291-4.

21. Rafiee M, Bara M, Stephens CP, Blackall PJ. Application of ERIC PCR for the comparison of isolates of Haemophilus parasuis. Aust Vet J [Internet]. 2000 Dec [cited 2012 Sep 30];78(12):846-9. Available from: http://doi.wiley.com/10.1111/j.1751-0813.2000.tb10507.x

22. Li J, Jiang P, Wang Y, Li Y, Chen W, Wang $X$, et al. Genotyping of Haemophilus parasuis from diseased pigs in China and prevalence of two coexisting virus pathogens. Prev Vet Med [Internet]. 2009 Oct 1 [cited 2012 Aug 2];91(2-4):274-9. Available from: http://dx.doi.org/10.1016/j.prevetmed.2009.06.004

23. Palzer A, Ritzmann M, Wolf $G$, Heinritzi K. Associations between pathogens in healthy pigs and pigs with pneumonia. Vet Rec [Internet]. 2008 Mar 1 [cited 2012 Aug 2];162(9):267-71. Available from: 
http://veterinaryrecord.bmj.com/content/162/9/267.full

24. Falk K, Lium B, Ødegaard Ø. Occurence of lung lesions and antibodies to serotypes 2 and 6 of Actinobacillus pleuropneumonia and to Haemophilus parasuis in 5176 slaughter pigs from 113 elite herds in Norway. In: Proceedings of the 11th International Pig Veterinary Society Congress. Lausanne; 1990. p. 31.

\section{Figures}

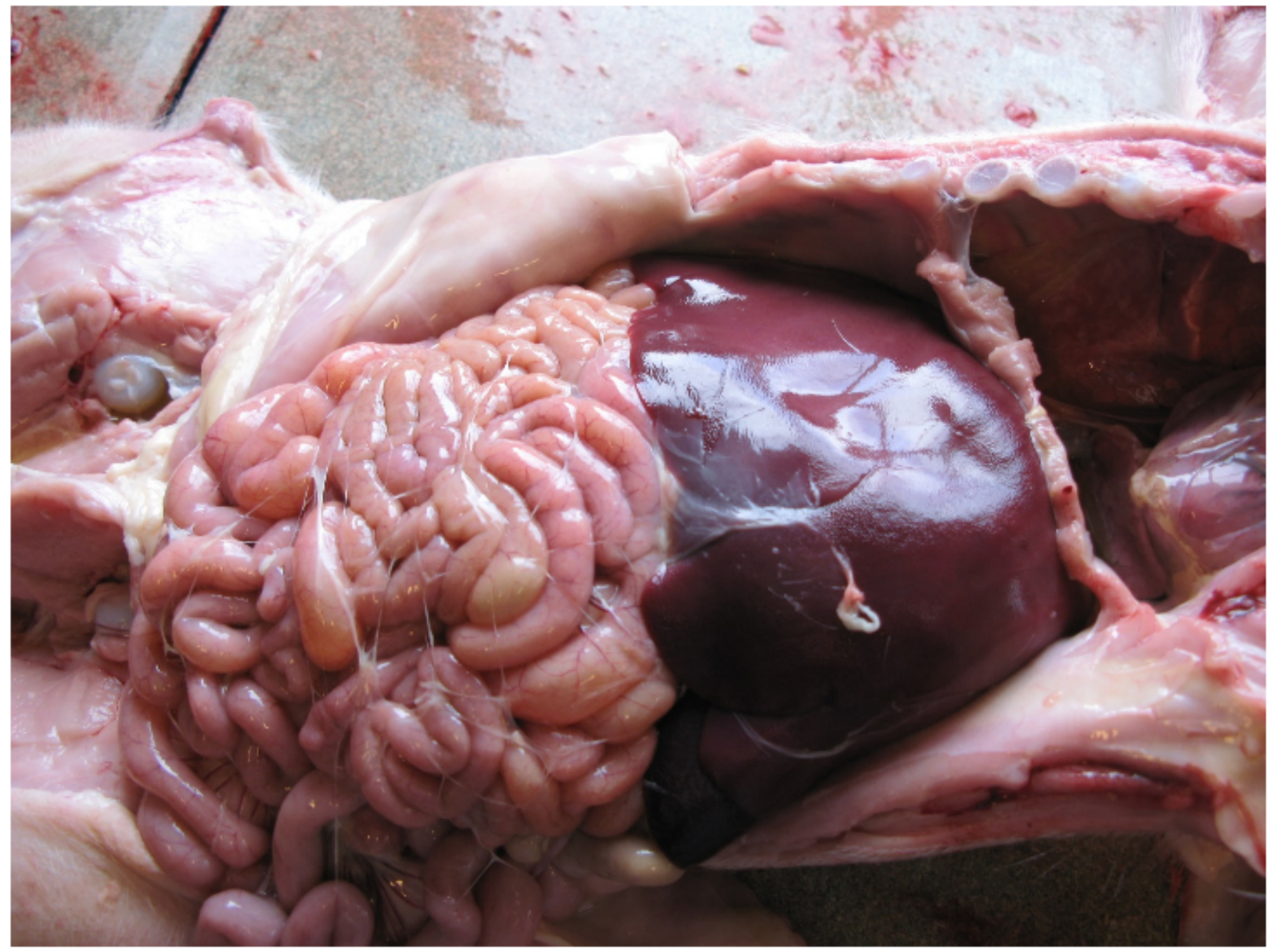

\section{Figure 1}

Gross pathology findings from on farm post-mortem examinations showing pale musculature, fine treads of fibrin over the intestines and fibrin membrane on liver capsule. 


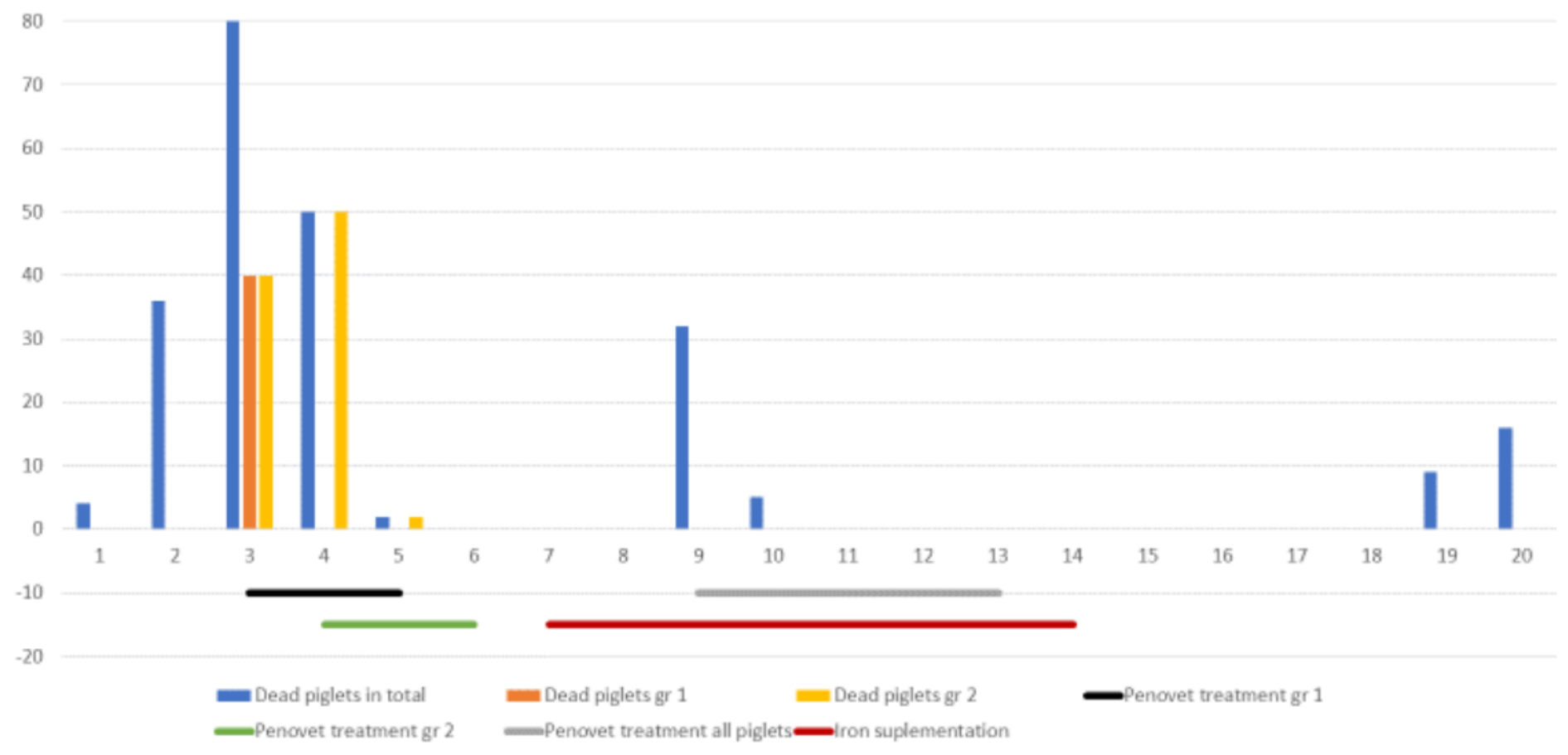

\section{Figure 2}

Number of dead piglets per day and the time of the different treatments 